\title{
Time-clustering behavior in the sequence of the aftershocks of the Al-Hoceima (Morocco) 24 February 2004 earthquake
}

\author{
L. Telesca ${ }^{1}$, M. Rouai ${ }^{2}$, and T. E. Cherkaoui ${ }^{3}$ \\ ${ }^{1}$ Istituto di Metodologie per l'Analisi Ambientale, CNR, Tito Scalo (PZ), Italy \\ ${ }^{2}$ Laboratory of Enginnering Geology, Faculty of Sciences, Meknes Universiy, P.O. Box 11201, Zitoune, Meknes, Morocco \\ ${ }^{3}$ Institut Scientifique, Mohamed V - Agdal University, Rabat, Morocco
}

Received: 3 August 2009 - Accepted: 30 September 2009 - Published: 8 December 2009

\begin{abstract}
The time dynamics of the aftershock sequence of the Al-Hoceima (Morocco) earthquake of 24 February 2004 has been investigated. The sequence of the occurrence times of the events with threshold magnitude $M_{t h} \geq 3.2$ is characterized by a time-clustering behavior, identified using different fractal methods (Fano Factor, Allan Factor, Count-based Periodogram), well suited to reveal scaling features in point processes. The obtained results not only show the presence of memory phenomena and correlation structures in the AlHoceima aftershocks, but also furnish quantitatively the estimate of the magnitude of such correlation by means of the estimate of the scaling exponent $\alpha$.
\end{abstract}

\section{Introduction}

Analyzing the temporal distribution of seismic sequences plays an important role in the general framework of the studies devoted to seismicity. The time-correlation structures governing observational time series can give useful information on the dynamical features of seismic processes and on the involved geodynamical mechanisms. In particular analyzing the temporal distribution of earthquakes could have great implications to improve the probabilistic techniques for seismic hazard analysis (Main, 1995, 1996).

Seismic processes display a scale-invariant behavior in several statistical features. Gutenberg and Richter (1944) found that earthquake size follows a power-law distribution. Scale-invariant features were determined in Kagan (1992) and Kagan and Jackson (1991); Kagan (1994) reviewed experimental evidences for earthquake scale-invariance. A theory to explain the presence of scale-invariance was proposed by Bak et al. (1988) in a pioneer work; they introduced the idea of self-organized criticality (SOC) starting from a

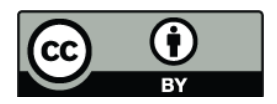

Correspondence to: L. Telesca

(luciano.telesca@imaa.cnr.it) simple cellular automaton model, namely sandpile. In this model, sand is dropped slowly into random locations on a lattice, one grain at a time. For a critical slope, when a new grain is added many avalanches are triggered. Numerically they obtained that the distribution of avalanche sizes presents a power-law spectrum.

The analysis of scaling laws concerning earthquakes has led to the development of a wide variety of physical models of seismogenesis used to better characterize the seismicity patterns (Sornette et al., 1990; Scholz, 1991).

Several distributions have been used to model seismic activity. The Poisson distribution implies the independence of each event from the time elapsed since the previous event. However, earthquake occurrence is characterized by clustering properties with both short and long timescales (Kagan and Jackson, 1991) that imply a temporal correlation among the seismic events. A complex phenomenon, like earthquakes, can be deeply known only if the different timescales governing its dynamics are well understood. Statistical approaches like the Fano Factor, Allan Factor, Count-based Periodogram, can furnish an exhaustive description of the temporal properties of point processes, and, in particular, earthquakes. Using those methodologies, the aftershock sequence of the Al-Hoceima (Morocco) 24 February 2004 earthquake is investigated.

Aftershock sequences, apparently related to the fault plane that slipped during the main event (Lay and Wallace, 1995), are the relevant characteristic of shallow earthquakes. An aftershock series displays temporal fractal properties, by means of the Omori's law (Utsu et al., 1995). The Omori's law gives only preliminary information of the timecorrelation structures that characterises the aftershocks of a main event. A deep investigation of these structures is provided by the analysis of second-order statistics. For this purpose, in this paper the aftershock sequence is modeled by a point process. A point process describes events that occur at some random locations in space and time, and it is

Published by Copernicus Publications on behalf of the European Geosciences Union. 
completely defined by the set of event times and hypocenter locations. Dividing the time axis into equally spaced contiguous counting windows of duration $\tau$, we produce a sequence of counts $\left\{N_{k}\right\}$, where $N_{k}$ represents the number of events falling into the $k$ th window. This sequence is a discreterandom process of non-negative integers. An important feature of this representation is that it preserves the correspondence between the discrete time axis of the counting process $\left\{N_{k}\right\}$ and the "real" time axis of the underlying point process, and the correlation in the process $\left\{N_{k}\right\}$ refers to correlation in the underlying point process (Thurner et al., 1997). Such a process may be called fractal when a number of relevant statistics exhibit scaling with related scaling exponents, that indicate the represented phenomenon contain clusters of points over a relatively large set of timescales (Thurner et al., 1997).

The temporal fluctuations of a process are investigated by the analysis of its power spectral density. The power spectral density provides a measure of how the power in a process is concentrated in various frequency bands, and for a process with scaling properties it follows a power-law behavior $S(f) \propto f^{-\alpha}$, with the exponent $\alpha$ measuring the strength of the cauterization. Of course, for a finite size fractal real process the power spectral density behaves as an inverse powerlaw function in a limited range of frequency, approaching an asymptotic value at high frequencies, at which the behavior of the process can be considered Poissonian. The clustering behavior of a point process leads to a power-law (fractal) shape of some of the statistics, used to describe its properties; we refer, in particular, to the Allan Factor (AF), that allows to estimate the spectral exponent $\alpha$; the numerical value of $\alpha$ is an indicator of the presence of clusterisation in the process (Thurner et al., 1997). If the point process is Poissonian, the occurrence times are uncorrelated; for this memoryless process $\alpha \approx 0$. On the other side, $\alpha \neq 0$ is typical of point processes with self-similar behavior; self-similar meaning that parts of the whole can be made to fit to the whole in some way by scaling (Mandelbrot, 1983).

\section{The methods}

The sequence of the recorded earthquakes can be expressed by a finite sum of Dirac's delta functions centered on the occurrence times $t_{i}$ :

$y(t)=\sum_{i=1}^{n} \delta\left(t-t_{i}\right)$

Dividing the time axis into equally spaced contiguous counting windows of duration $\tau$, we can produce a sequence of counts $\left\{N_{k}(\tau)\right\}$, with $N_{k}(\tau)$ denoting the number of earthquakes in the $k$-th window:

$N_{k}(\tau)=\int_{t_{k-1}}^{t_{k}} \sum_{j=1}^{n} \delta\left(t-t_{j}\right) d t$.
A measure, related to the variability of successive counts, useful to detect the event clustering in a point process, is the Allan Factor (AF) (Allan, 1966), defined as the variance of successive counts for a specified counting time $\tau$ divided by twice the mean number of events in that counting time

$\mathrm{AF}(\tau)=\frac{<\left(N_{k+1}(\tau)-N_{k}(\tau)\right)^{2}>}{2<N_{k}(\tau)>}$.

This measure reduces the effect of possible nonstationarity of the point process, because it is defined in terms of the difference of successive counts. The AF of a fractal point process varies with the counting time $\tau$ with a power-law form:

$\mathrm{AF}(\tau)=1+\left(\frac{\tau}{\tau_{1}}\right)^{\alpha}$

with $0<\alpha<3$ over a large range of counting times $\tau$ (Lowen and Teich, 1995 ). The monotonic power-law increase is representative of the presence of fluctuations on many timescales. The scaling exponent $\alpha$ is the so-called fractal exponent. If $\alpha>0$ then the represented phenomenon contains clusters of points over a relatively large set of timescales. If $\alpha \approx 0$, the earthquake occurrence process is Poissonian and the occurrence times are uncorrelated. The crossover timescale $\tau_{1}$ is the fractal onset time, and marks the lower limit for significant scaling behavior in the AF (Teich et al., 1996), so that for $\tau \ll \tau_{1}$ the clustering property becomes negligible at these timescales. Thus, $\tau_{1}$ is estimated as the timescale over which the AF increases as a power-law function of the timescale $\tau$. AF assumes values near unity for Poisson processes.

The count-based Periodogram (PG) (Teich et al., 1996), that is the periodogram of the sequence of the counts, is another statistical measure that allows estimating the fractal exponent $\alpha$. This measure performs an estimate of the power spectral density, which gives information on how the power of the process is concentrated at various frequency bands. The calculation of the PG by means of a count-based approach implies the division of the total observation period into a number $N$ of no overlapping windows of length $w$. The sequence of the counts is then Fourier transformed and the PG is obtained by the squares of the coefficients of the Fourier representation of the series of counts. Generally, in order to use the Fast Fourier Transform (FFT) the number $N$ of the windows of duration $w$ is a power of two. For point processes with scaling properties the PG decreases as a power-law function of the frequency $f$ over a significant range of frequencies, $S(f) \propto f^{-\alpha}$. Of course, for a finite size fractal real process the power spectral density behaves as an inverse power-law function in a limited range of frequencies, approaching an asymptotic value at high frequencies, at which the behavior of the process can be considered Poissonian. The numerical value of $\alpha$ is an indicator of the presence of clusterization in the process (Thurner et al., 1997). If the point process is Poissonian, the occurrence times are 


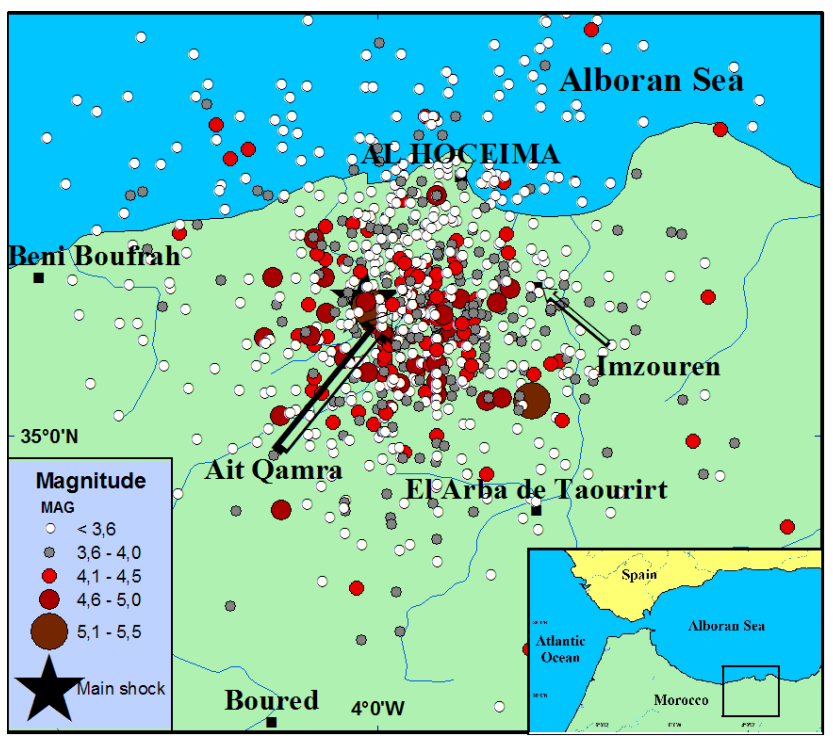

Fig. 1. Hypocenter of 938 aftershocks recorded with a minimum of five stations.

uncorrelated; for this memoryless process, the PG is approximately flat for any frequency bands and $\alpha \approx 0$. On the other side, $\alpha \neq 0$ is typical of point processes with scaling behavior. This method introduces a bias at higher frequencies, since the fine time resolution information is lost as a result of the minimum count-window size. But, the estimation of the exponent $\alpha$ principally involves lower frequencies where this bias is negligible.

\section{Analysis of Al-Hoceima aftershock series}

Al-Hoceima region (NE Morocco, Fig. 1) is the most seismically active zone of the country (Cherkaoui, 1991). It is located in the eastern Mediterranean collision boundary between African and Eurasian plates. The average displacement is about $5 \mathrm{~mm} / \mathrm{y}$ (Argus et al., 1989). This convergent movement of Africa towards Eurasia induces high and diffuse seismicity, with a strike-slip dominant mechanism. On 24 February 2004 at 2 h $27 \mathrm{mn} 47$ s, Northern Morocco was again struck by a shallow earthquake of magnitude $M_{w}=6.3$ (Stich et al., 2005) and intensity IX (MSK scale).The earthquake occurred in Ait Qamra, a village located $15 \mathrm{~km}$ southwest of Al-Hoceima. The earthquake shook the region, causing the considerable damages to the Ait Qamra, Imzouren and its surrounding regions. The toll of earthquake is catastrophic: 629 dead, 926 injured, 15000 of people lost their homes and more than 2500 houses destroyed. The main shock was located at $35.129^{\circ} \mathrm{N}-4.011^{\circ} \mathrm{W}$ and depth $=5.5 \mathrm{~km}$. Investigations carried out rapidly in the region just after the earthquake resulted in serious damages to the constructions: hoses, schools, mosques (Cherkaoui, 2005). The earthquake main event was followed by a long aftershock sequence dur-

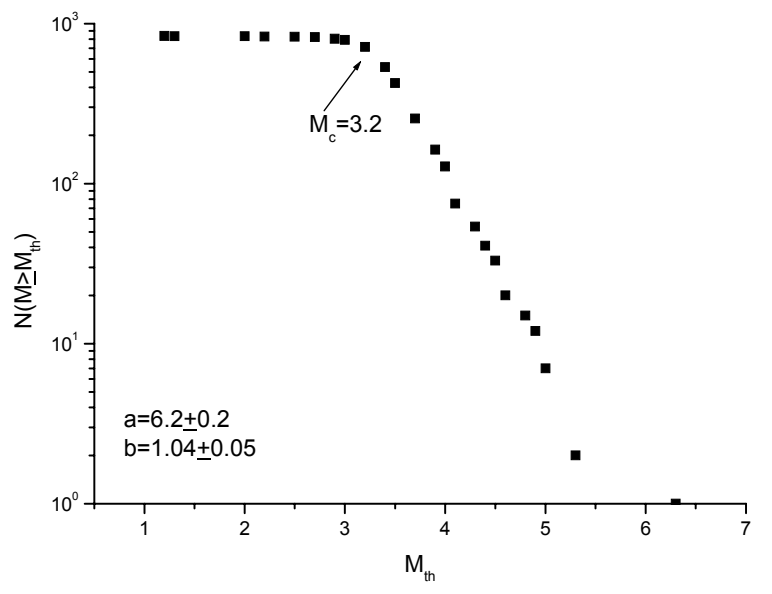

Fig. 2. Gutenberg-Richter analysis of the aftershock sequence. The minimum magnitude of completeness is 3.2. The b-value, estimated as the slope of the line fitting the cumulative number of events versus threshold magnitude, for $M_{t h} \geq 3.2$ is 1.04 .

ing the time from 24 February to 31 May. During this period six strong aftershocks occurred with $m_{b} \geq 5$.0. A total of 838 shocks have been identified as having occurred in the neighbourhood of the main shock and recorded with of a minimum of five stations. These events were located using Hypo71PC program (Lee and Valdés., 1985) with the $P$ and $S$ arrival times collected at all the available stations (Fig. 1).

In order to study the temporal distribution of a complete catalogue, we performed the Gutenberg-Richter analysis (Fig. 2), obtaining a b-value of 1.04 for the cumulative distribution of events with magnitude $M \geq 3.2$, that can be considered the minimum magnitude of completeness. We computed the $\operatorname{AF}(\tau)$ for counting times of duration of $50 \mathrm{~s}$ to $\mathrm{T} / 10$, where $T$ represents the total observation period; the upper cut-off is due to the finite data length (Thurner et al., 1997) . The AF plot (Fig. 3) gives clear indication of the fractal behaviour; the AF is seen to increase with linear behaviour for counting times greater than approximately $\tau_{1} \sim 10^{4} \mathrm{~s}$ in bilogarithmic scales, which can give a rough estimate of the fractal onset time. The slope of the straight line that fits the $\mathrm{AF}$ in the linear range gives the estimate of the exponent $\alpha$. In particular we estimated $\alpha \sim 0.9$. Figure 4 shows the results obtained performing the PG analysis; the estimated scaling exponent $(\alpha \sim 1.09)$ and the crossover timescale are consistent with those calculated by the AF method.

\section{Conclusions}

The occurrence time sequence of aftershocks of the AlHoceima (Morocco) 24 February 2004 earthquake has been studied by means of quantitatively fractal tools (AF and PG). These methods, extensively used in other scientific fields, are very useful to reveal the presence of clustering behavior in 


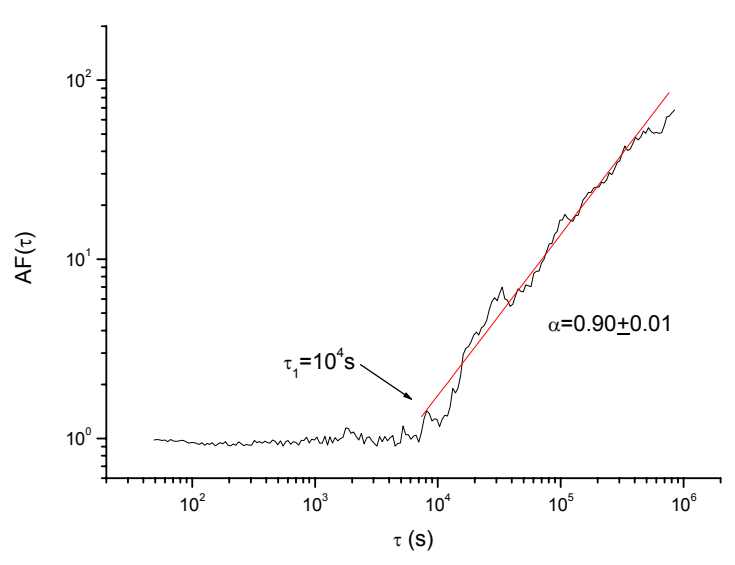

Fig. 3. Doubly logarithmic plot of the Allan Factor curve versus the counting time $\tau$ for the aftershock sequence, with threshold magnitude $M_{t h}=3.2$.

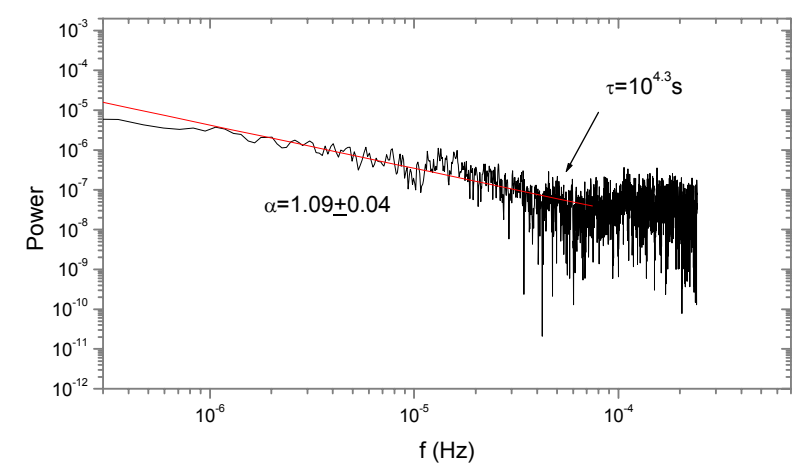

Fig. 4. Doubly logarithmic plot of the Count-based Periodogram versus the frequency $\mathrm{f}$ for the aftershock sequence, with threshold magnitude $M_{t h}=3.2$.

time series represented as realizations of point processes, and to determine, for the point process under investigation, the exponent of its power spectral density, which behaves like a power-law function if governed by time-clustering properties.

Time-occurrence sequences of Al-Hoceima 24 February 2004 aftershocks characterized by magnitude greater or equal to 3.2 show the presence of power-law behavior in the performed statistics, indicating the existence of fluctuations on many timescales and therefore of fractal clustering.

Acknowledgements. This study was supported by the bilateral project Italy-Morocco in the frame of the CNR/CNRST bilateral agreement for scientific cooperation.

Edited by: M. E. Contadakis

Reviewed by: J. Sebbani and another anonymous referee

\section{References}

Allan, D. W.: Statistics of atomic frequency standards, Proc. IEEE, 54, 221-230, 1966.
Argus, D. F., Gordon, F. G., De Mets, G., and Sten, S.: Closure of Africa-Eurasia-North America plate motion and tectonics of Gloria fault, J. Geophys. Res. , 94(B5), 5585-5602, 1989.

Bak, P., Tang, C., and Wiensenfeld, K.: Self-organizing criticality, Phys. Rev. A, 38, 364-374, 1988.

Cherkaoui, T. E.: Contribution à l'étude de l'aléa sismique au Maroc, Ph.D. thesis, University of Grenoble, France, 246 pp., 1991.

Cherkaoui, T. E.: Le séisme d'Al Hoceima du 24 février 2004, in: Trav. Col. Nat. Sur : Région de Taza-Al Hoceima-Taounate, ressources et stratégies de développement, Pub. Fac. Polydisciplinaire de Taza, no 1, 27-38, 2005.

Gutenberg, B. and Richter, C. F.: Frequency of earthquakes in California, B. Seismol. Soc. Am., 34, 185-188, 1944.

Kagan, Y. Y.: Correlations of earthquake focal mechanism, Geophys. J. Int., 110, 305-320, 1992.

Kagan, Y. Y. and Jackson, D. D.: Long-term earthquake clustering, Geophys. J. Int., 104, 117-133, 1991.

Kagan, Y. Y.: Observational evidence for earthquakes as a nonlinear dynamic process, Physica D, 77, 160-192, 1994.

Lay, T. and Wallace, T. C.: Modern Global Climatology, Academic, San Diego, California, 1995.

Lee, W. H. K. and Valdés, C. M.: HYP071PC: A personal computer version of the HYPO71 earthquake location program, US Geological Survey Open File Report 85-749, 43 pp., 1985.

Lowen, S. B. and Teich, M. C.: Estimation and simulation of fractal stochastic point processes, Fractals, 3, 183-210, 1995.

Main, I. G.: Earthquakes as critical phenomena: implications for probabilistic seismic hazard analysis, B. Seimol. Soc. Am., 85(5), 1299-1308, 1995.

Main, I. G.: Statistical physics, seismogenesis, and seismic hazard, Rev. Geophys., 34, 433-462, 1996.

Mandelbrot, B. B.: The Fractal Geometry of Nature, W. H. Freeman, New York, 1983.

Scholz, C. H.: Earthquakes and Faulting: self-organized critical phenomena with characteristic dimension, in: Spontaneous formation of space-time structures and criticality, edited by: Triste, T. and D. Sherrington, D., Kluwer Academic Publishers, Netherlands, 41-56, 1991.

Sornette, D., Davy, P., and Sornette, A.: Structuration of the lithosphere in plate tectonics as a self-organized critical phenomena, J. Geophys. Res., 95, 17353-17361, 1990.

Stich, D., Mancilla, F., Baumont, D., and Morales, J.: Source analysis of the Mw 6.32004 Al Hoceima earthquake (Morocco) using regional apparent source time functions, J. Geophys. Res., 110, B06306, 2005.

Teich, M. C., Heneghan, C., Lowen, S. B., and Turcott, R. G.: Estimating the fractal exponent of point processes in bilogical systems using wavelet- and Fourier-transform methods, in: Wavelets in Medicine and Biology, edited by: Aldroubi, A. and Unser, M., CRC Press, Boca Raton, FL, 383-412, 1996.

Thurner, S., Lowen, S. B., Feurstein, M. C., Heneghan, C., Feichtinger, H. G., and Teich, M. C.: Analysis, Synthesis, and Estimation of Fractal-Rate Stochastic Point Processes, Fractals, 5, 565-596, 1997.

Utsu, T., Ogata, Y., and Matsu'ura, R. S.: The centenary of the Omori formula for a decay law of aftershock activity, J. Phys. Earth, 43, 1-33, 1995. 